Desarrollo Humano, Democracia y Ciudadanía

En la sección de Desarrollo Humano, Democracia y Ciudadanía se presentan cuatro artículos relacionados con educación, posconflicto, alfabetización digital y obsolescencia. El primero se titula "Educación para la ciudadanía en el posacuerdo" de María Liliana Días Perdomo y Nelson Darío Rojas Suárez. Este artículo de revisión tiene como objetivo ubicar el papel que ha tenido la educación en el posconflicto en países que han vivido algún proceso de paz. Afirman que mientras la educación conserve parámetros violentos y autoritarios no será posible crear una cultura de paz.

El segundo artículo de esta sección, "La alfabetización digital en la formación de competencias ciudadanas en la básica primaria" de los autores Gerzon Yair Calle Álvarez y Angelina Lozano Prada, describe las posibilidades que ofrece implementar una propuesta didáctica de alfabetización digital en la formación de competencias ciudadanas. La metodología utilizada en este estudio fue investigativa-descriptiva. Los autores concluyen que es necesario desarrollar competencias instrumentales, cognitivas y sociales en una propuesta didáctica de alfabetización digital para la formación de competencias ciudadanas.

El último artículo del profesor Gregorio Hernández Pulgarín y Manuel Felipe García Arias titulado "Obsolescencia y revitalización de un parque: acción colectiva, cognición y ciudadanía en la apropiación del espacio público” muestra el análisis hecho por los autores acerca de las transformaciones de un parque en Manizales-Colombia a fin de mostrar cómo la acción colectiva que se materializa en el uso que se le da y algunos procesos cognitivos que permiten representarlo, influyen en la manera en que es apropiado. Afirman que los espacios públicos son escenarios de confluencia compleja y conflictiva, dado que dependiendo de los usuarios y el uso que estos le den al espacio, se pueden generar diferentes formas de apropiación.

\title{
Diversidad y Justicia Social
}

En la sección de Diversidad y Justicia Social se incluyeron cuatro artículos. El primero se titula "Investigación para la paz: perspectiva de paz desde la primera infancia y sus agentes relacionales” de Estefanía Aristizábal Ramírez. Tiene como objetivo ampliar los horizontes reflexivos en torno a una crianza para la paz en tiempos de posconflicto para los niños de la primera infancia a partir del agenciamiento social de los docentes y cuidadores. El estudio se realizó en el municipio de Marmato-Caldas y el enfoque metodológico fue cualitativo y crítico social. 
Por su parte, Elizabeth Torres presenta el artículo de revisión "Tramas del reclutamiento y participación de niños en el conflicto armado colombiano”. Su objetivo es el análisis de la trama narrativa de la vinculación y participación de niños en los grupos armados colombianos. La autora, a partir de lo analizado, reconoce que el fenómeno de la vinculación de los niños a grupos armados no se debe solo al conflicto, sino que también es consecuencia del abandono del Estado a los menores.

El objetivo de Henry Torres Vásquez con su artículo "La operación Cóndor y el terrorismo de Estado” es analizar la realidad histórica de las actividades criminales efectuadas por integrantes de las fuerzas militares desde 1975 en algunos países de Suramérica y su persecución, desaparición y tortura hacia presuntos terroristas y comunistas. Argumenta que para evitar y eliminar este tipo de prácticas es necesaria la intervención de tribunales internacionales, de tal forma que se pueda evitar la impunidad frente a este tipo de crímenes.

El último artículo de esta sección se titula "La posesión frente al derecho de propiedad: un debate sobre vigencia y pertinencia sin resolver" escrito por Roberto Silva Fernández, tiene como objetivo llamar la atención sobre la justificación histórica y jurídica de la posesión, que como derecho provisional conserva vigencia casi prevalente sobre el derecho de propiedad en el sistema jurídico colombiano. Concluye que en tiempos en que el concepto de la propiedad y su función social se ha reevaluado y ampliado, resulta obsoleto que conserve vigencia el derecho de posesión, en cuanto desvirtúa un derecho que ha sido fundamental en las democracias occidentales como es el de la propiedad.

Enfoques en Trabajo Social: Investigación y Práctica Profesional

El primer artículo de esta última sección se titula "Más allá de la caridad y la filantropía: el surgimiento del Trabajo Social en Antioquia” escrito por Diana Ramírez Duarte, Leidy Karina López Palacio y Daniela Uribe Espinosa. Es un estudio de la historia del Trabajo Social en el Departamento de Antioquia en Colombia. Las autoras afirman que esta profesión se dio del interés de una parte de la clase burguesa antioqueña para intervenir las expresiones de las cuestiones sociales causados por el capitalismo, lo que se esperaría mantendría el orden social y generaría condiciones favorables para la reproducción y división de la fuerza de trabajo.

Luego está el artículo Contextos, tendencias y actores de la Reconceptualización del profesor Sergio Quintero Londoño. Su objetivo es demostrar las mediaciones entre el desarrollo sociohistórico del modo de producción capitalista y el Trabajo Social, entendida como una profesión inscrita en la división socio/técnica del trabajo. El autor afirma que la Reconceptualización es un movimiento que hace parte de la renovación profesional del Trabajo Social, en la que se encuentran tendencias teóricas/políticas diferenciadas. 
El último artículo de esta sección "Funciones de los Trabajadores Sociales del área de la salud en los hospitales y clínicas de tercero y cuarto nivel de la ciudad de Medellín-Colombia” escrito por Marta Elena Correa Arango, Alejandra Corena Argota, Camila Chavarriaga Estrada, Karla García Valencia y Sara Usme Baena, tiene como objetivo dar a conocer, a través de un estudio cualitativo-descriptivo, las funciones desarrolladas por los Trabajadores Sociales en el ámbito de la salud, específicamente en la ciudad de Medellín. Como resultado del trabajo de investigación, los autores argumentan que los trabajadores sociales tienen una perspectiva integral de la salud, lo que permite que su participación en este campo sea integral y sistémica con los pacientes y sus redes de apoyo familiares y sociales. Por lo tanto, estos profesionales cumplen un significativo papel integrador en equipos interdisciplinarios, lo que contribuye a la accesibilidad e integralidad del servicio de salud. 